Introduction: The aim of the present study was to evaluate the changes in height, weight and in the prevalence of overweight and obesity between 1998 and 2007 in Cameroonian children.

Method: Two community-based cross-sectional studies were conducted in Cameroon in 1998 (rural, $n$ 825; urban, $n$ 675) and 2007 (rural, $n$ 1427; urban, $n$ 625) in children aged 5-16 years. Body weight $(\mathrm{kg})$ and height $(\mathrm{m})$ were measured, and BMI was calculated $\left(\mathrm{kg} / \mathrm{m}^{2}\right)$. The prevalence of overweight and obesity was determined according to the genderand age-specific cut-offs of the new international reference (International Obesity Task Force).

Results: There was a slight but significant difference in the age of participants between the two study periods. The age- adjusted height, weight and BMI increased significantly from 1998 to 2003 in rural and urban areas. This increase was higher in the rural area for height and weight. In rural area, the prevalence of overweight + obesity increased from $4 \cdot 6 \%$ to $10 \cdot 9 \%(P<0 \cdot 001)$ in girls and from $3.3 \%$ to $7 \cdot 3 \%(P<0 \cdot 02)$ in boys. In urban area, the prevalence of overweight + obesity increased from $7 \cdot 3 \%$ to $11 \cdot 4 \%$ in girls and from $3.0 \%$ to $9 \cdot 3 \%$ in boys. However, in urban area, the increase reached the significance level only in boys $(P<0 \cdot 001)$.

Conclusions: Over a 10-year period, there was an increase in height and BMI in Cameroonian boys and girls. These results show that the increase in the prevalence of adiposity in Cameroon is accompanied by a global trend of accelerated growth, particularly marked in rural area.

\title{
11 - The factors affecting obesity
}

\section{Sofokleous, G Sofokleous and C Theocharides}

National and Kapodistrian University of Athens

Introduction: Obesity in primary educational level is generally accepted as one of the most serious problems, due to its effects on the long-term complications of physical and mental health. The purpose of the present study was to examine the factors that affect obesity of the students aged 9-12 years of two primary schools in Nicosia, in order to bring the problem under control by shifting their diet towards healthy solutions and exercise.

Materials/method: A total of 100 pupils of two primary schools of Nicosia (urban and rural area) were randomly selected to participate by answering a questionnaire of personal characteristics, socio-economic status and eating habits. Anthropometric data were obtained (height and weight) in order to calculate BMI and categorized children to (i) normal
BMI, (ii) overweight children and (iii) obese children. SPSS package was used for the data analysis and proceeding.

Results: Children with more hours of television viewing were found to have higher levels of BMI. Unhealthy nutrition habits had a positive correlation with BMI; however, physical exercise had a negative correlation. No statistically significant difference was found between sex, age, place of residence, socio-economic status and children's BMI.

Conclusions: The results of this research are similar with other published reports showing that the most important factors affecting obesity of children aged 9-11 years in two elementary schools in Nicosia, are exercising, eating habits and television viewing. Contributions should be done, based on these results, for further investigations.

\section{2 - Secular trends of overweight and obesity in Polish schoolchildren from rural area}

\author{
A Mazur ${ }^{1}$, E Małecka-Tendera ${ }^{2}$ and K Klimek ${ }^{2}$ \\ ${ }^{1}$ Institute of Physiotherapy, University of Rzeszów, Poland: ${ }^{2}$ Medical University of Silesia, Katowice, Poland
}

Aim: The prevalence of overweight in children has markedly increased over the past few decades worldwide. The study aim was to monitor 10 years' trend for childhood overweight/ obesity prevalence in schoolchildren from rural area in South-East Poland. BMI mean values were significantly higher in girls' group studied in 1998 compared with 2008 group. For individual age groups mean BMI values of girls studied in 1998 were significantly higher than those of girls studied in 2008 aged 10-14 years. Prevalence of overweight in boys in the 2008 study was significantly higher in 